CONGENITAL HEART DISEASE

\title{
Transcatheter closure of high pulmonary artery pressure persistent ductus arteriosus with the Amplatzer muscular ventricular septal defect occluder
}

B D Thanopoulos, G S Tsaousis, M Djukic, F Al Hakim, N G Eleftherakis, S D Simeunovic

Heart 2002;87:260-263

Background: The design of devices currently used for closure of persistent ductus arteriosus (PDA) with high pulmonary artery pressure is not ideal and there is a risk of embolisation into the aorta.

Objective: To investigate the use of the Amplatzer muscular ventricular septal defect occluder (AMVSDO) for treatment of PDA with high pulmonary artery pressure.

Patients and design: Seven patients, aged 5-12 years, with large PDAs and systemic or near systemic pulmonary artery pressure underwent attempted transcatheter closure using the AMVSDO. The device consists of two low profile disks made of a nitinol wire mesh with a $7 \mathrm{~mm}$ connecting waist. Balloon occlusion of the duct was performed before closure from the venous side, and prosthesis size was chosen according to the measured diameter of the occluding balloon. A 7 French sheath was used to

See end of article for authors' affiliations

Correspondence to: Dr Vasilios D Thanopoulos, Department of Cardiology, Aghia Sophia Children's Hospital, Thivon and Levadias 1, Athens 11527, Greece;

vasiliosthanopoulos@usa.net

Accepted

13 November 2001 deliver the device. All patients underwent a complete haemodynamic and angiographic study one year after occlusion.

Results: The mean (SD) angiographic PDA diameter was 9.8 (1.7) mm (range 7-13 mm) and the mean AMVSDO diameter was $11.4(1.8) \mathrm{mm}$ (range 9-16 mm). $Q_{p} / Q_{s}$ ranged from 1.9-2.2 (mean $2.0(0.1))$. Successful device delivery and complete closure occurred in all patients $(100 \%$ occlusion rate, $95 \%$ confidence interval $59.04 \%$ to $100.00 \%$ ). Mean systolic pulmonary artery pressures were as follows: before balloon occlusion, 106 (13) mm Hg; during occlusion, 61 (6) mm Hg; immediately after the procedure, $57(5) \mathrm{mm} \mathrm{Hg}$; and at the one year follow up catheterisation, 37 (10) $\mathrm{mm} \mathrm{Hg}$. Fluoroscopy time was 10.4 (4.3) min (range 7-18 min). No complications occurred.

Conclusions: AMVSDO is an important adjunct for closure of large PDAs associated with high pulmonary artery pressure. Further studies are required to document its efficacy, safety, and long term results in a larger number of patients.
$\mathrm{T}$ ranscatheter closure of persistent ductus arteriosus (PDA) using various occluders and coils is a well established method. ${ }^{1-7}$ However, not all patients with PDA are amenable to this type of treatment, as these devices-including the new Amplatzer duct occluder ${ }^{8-10}$ - are not suitable for large high pulmonary artery pressure ducts (HPAP-PDA). In the presence of high pulmonary artery pressure such devices carry the risk of embolising into the aorta. The Amplatzer muscular ventricular septal defect occluder (AMVSDO) is a new device which has recently been used successfully for transcatheter closure of muscular ventricular septal defects. ${ }^{11}{ }^{12}$ This occluder, which is available in a variety of sizes, may be more suitable for use with HPAP-PDA as its double disk tends to anchor the device, preventing embolisation into the aorta. In this study we report the successful use of the AMVSDO for treating HPAP-PDA.

\section{METHODS}

\section{Device design}

The AMVSDO (AGA Medical Corporation, Golden Valley, Minnesota, USA) has been described in detail in previous reports. ${ }^{1{ }^{12}}$ In brief, the AMVSDO is a self centering and repositionable device constructed of 0.004 inch $(0.1 \mathrm{~mm})$ Nitinol wires, tightly woven into two flat round discs with a $7 \mathrm{~mm}$ connecting waist. The left disc is $4 \mathrm{~mm}$ larger than the waist and the right disc is $3 \mathrm{~mm}$ larger than the waist. Prostheses are currently available in sizes (waist diameters) ranging from 4-24 mm. The device is delivered through a long 6-8 French sheath.

\section{Patient population}

From May 1998 to August 2000, seven patients with clinical and echocardiographic findings of a large PDA and pulmonary hypertension underwent transcatheter closure with the AMVSDO. Their median age was 9 years (range 5-12 years) and their median body weight was $26 \mathrm{~kg}$ (19-57 kg). Three patients had symptoms of heart failure or failure to thrive. On cross sectional and Doppler echocardiography there was evidence of bidirectional shunting through the PDA with left atrial and left ventricular enlargement.

Informed parental consent for the procedures was obtained in each patient.

\section{Procedure}

The technique of transcatheter closure of PDA using the AMVSDO was similar to that described by Masura and colleagues with the use of the Amplatzer duct occluder. ${ }^{8}$ After percutaneous puncture of the femoral artery and vein, a complete haemodynamic evaluation was performed with pressure and saturation measurements taken in all cardiac chambers. A biplane descending aortogram in anteroposterior and lateral projections was performed with a 5 or 6 French pigtail catheter to define the size and anatomy of the PDA (fig 1, panel 1) Pulmonary artery and aortic pressure measurements were repeated during balloon duct occlusion, which was performed from the venous side using an 8 French Berman angiographic catheter or a Meditech sizing balloon (Meditech, Watertown, Massachusetts, USA). In addition to echocardiographic evidence (left atrial and left ventricular enlargement)

Abbreviations: AMVSDO, Amplatzer muscular ventricular septal defect occluder; HPAP-PDA, high pulmonary artery pressure ducts; PDA, persistent ductus arteriosus 

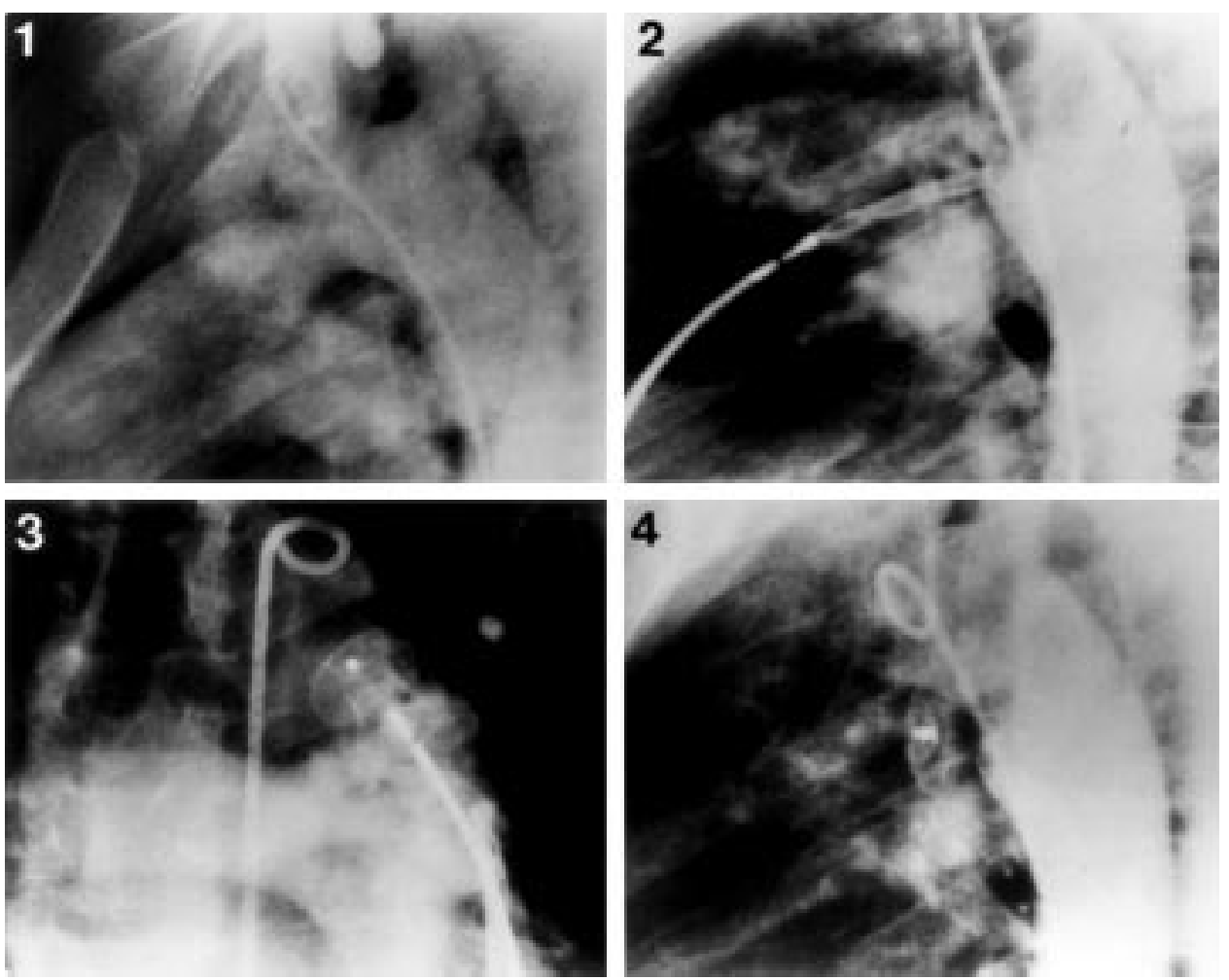

Figure 1 Steps in the transcatheter closure technique with the Amplatzer muscular ventricular septal defect occluder (AMVSDO) in an 11 year old patient with a large high pulmonary artery pressure persistent ductus arteriosus (PDA) (patient 3). (1) Descending aortogram in the lateral projection showing a large PDA measuring $10 \mathrm{~mm}$ in diameter. (2) Deployment of the retention disc of a $12 \mathrm{~mm}$ AMVSDO opposite the orifice of the duct. (3) Pulmonary arteriogram in the cranial projection showing good position of the device with no obstruction of the pulmonary artery. (4) Descending aortogram 10 minutes after release of the device, revealing complete closure with good device position.

and haemodynamic findings (ratio of pulmonary to systemic blood flow $\left.\left(\mathrm{Q}_{\mathrm{p}} / \mathrm{Q}_{\mathrm{s}}\right) \geqslant 1.9: 1\right)$ indicating a significant left to right shunt, a fall in the systolic pulmonary artery pressure of more than $30 \%$ during balloon occlusion was our criterion for proceeding to transcatheter closure. Subsequently, a 5 French Cobra type catheter was advanced percutaneously from the venous side through the PDA into the descending aorta. Using an exchange $260 \mathrm{~cm}, 0.035$ inch guide wire, the cobra catheter was exchanged for a 7 French delivery sheath which was advanced directly through the femoral vein and positioned in the proximal descending aorta.

An appropriately sized occluder (waist diameter equal to the balloon stretched PDA diameter) was screwed to the delivery cable, pulled into the loader, and introduced into the guiding sheath. Under fluoroscopic guidance, the occluder was advanced into the descending aorta, where the left disk was deployed and pulled gently against the orifice of the duct (fig 1, panel 2). Correct position was confirmed by injection of contrast medium through the aortic catheter into the descending aorta. Using gentle tension on the delivery cable, the sheath was pulled back to deploy the rest of the device. With the device still attached to the delivery cable, cross sectional colour Doppler echocardiography, pulmonary arteriography (fig 1, panel 3), and descending aortography (hand injection of contrast medium) were done to confirm proper device position and exclude left pulmonary or aortic obstruction. Once optimal position was confirmed, the AMVSDO was released by counterclockwise rotation of the delivery cable. A repeat aortogram (fig 1, panel 4) and a complete haemodynamic evaluation were performed to check for residual shunts and change of pressures. Prophylactic antibiotics were not routinely given during the procedure. All patients were sent home 24 hours after the procedure on no drug treatment. Endocarditis prophylaxis was discontinued at the 12 month follow up visit if the duct was completely closed.

\section{Follow up}

A chest $x$ ray and complete cross sectional and colour Doppler echocardiographic studies were performed on all patients at 24 hours, one month, and serially at 3-6 month intervals. In addition, all patients underwent a complete haemodynamic and angiographic study one year after the transcatheter closure.

\section{Statistical analysis}

Results were analysed with the Stratgraphic statistical program and expressed as mean (SD), with confidence intervals where applicable. Preocclusion and postocclusion data were compared using the paired Wilcoxon test. A probability value of $\mathrm{p}<0.05$ was considered significant.

\section{RESULTS}

The clinical and haemodynamic data before and after occlusion, and the outcome in the seven patients, are shown in table 1. According to Krichenko's PDA classification, ${ }^{13}$ five patients had type $\mathrm{A}$, one had type $\mathrm{C}$, and one had type $\mathrm{E}$. The length of the duct varied between 6-8 mm (mean 7.2 (0.5) $\mathrm{mm}$ ). The mean duct diameter (pulmonary end) was 9.8 (1.7) $\mathrm{mm}$ (range $7-13 \mathrm{~mm}$ ). The mean AMVSDO diameter was $11.4(1.8) \mathrm{mm}(9-16 \mathrm{~mm})$. The pulmonary to systemic flow ratio $\left(\mathrm{Q}_{\mathrm{p}} / \mathrm{Q}_{\mathrm{s}}\right)$ varied between 1.9-2.2 (mean $\left.2.04(0.14)\right)$. Patient 7 had a perimembranous ventricular septal defect $6 \mathrm{~mm}$ in size in addition to the PDA. All patients had systemic or near systemic systolic pulmonary artery pressure (mean 106 (13) $\mathrm{mm} \mathrm{Hg}$; mean systolic aortic pressure 112 (9) $\mathrm{mm} \mathrm{Hg}$ ). Device delivery was successful and associated with complete closure in all seven patients ( $100 \%$ closure rate, $95 \%$ confidence interval $59.04 \%$ to $100 \%$ ). There was a significant fall $(p<0.05)$ in mean systolic pulmonary artery pressure during balloon occlusion (to 6l (6) $\mathrm{mm} \mathrm{Hg}$ ) and immediately after the placement of the AMVSDO (to 57 (5) $\mathrm{mm} \mathrm{Hg}$ ). Fluoroscopy time was 10.4 (4.5) minutes (range 
Table 1 Clinical and haemodynamic data

\begin{tabular}{|c|c|c|c|c|c|c|c|c|c|c|c|c|}
\hline \multirow{2}{*}{$\begin{array}{l}\text { Patient } \\
\text { No/age } \\
\text { (years) }\end{array}$} & \multirow[b]{2}{*}{ Weight $(\mathrm{kg})$} & \multirow{2}{*}{$\begin{array}{l}\text { Duct } \\
\text { diameter } \\
(\mathrm{mm})\end{array}$} & \multirow{2}{*}{$\begin{array}{l}\text { AMVSDO } \\
\text { diameter } \\
(\mathrm{mm})\end{array}$} & \multicolumn{3}{|c|}{ Before closure } & \multicolumn{3}{|c|}{ After closure } & \multicolumn{3}{|c|}{ Follow up catheterisation } \\
\hline & & & & $\begin{array}{l}\text { SPAP } 1 \\
(\mathrm{~mm} \mathrm{Hg})\end{array}$ & $\begin{array}{l}\text { SPAP 2 } \\
(\mathrm{mm} \mathrm{Hg})\end{array}$ & $\begin{array}{l}\text { SAOP } \\
(\mathrm{mm} \mathrm{Hg})\end{array}$ & $\begin{array}{l}\text { SPAP } \\
(\mathrm{mm} \mathrm{Hg})\end{array}$ & $\begin{array}{l}\text { SAOP } \\
(\mathrm{mm} \mathrm{Hg})\end{array}$ & Results & $\begin{array}{l}\text { SPAP } \\
(\mathrm{mm} \mathrm{Hg})\end{array}$ & $\begin{array}{l}\text { SAOP } \\
(\mathrm{mm} \mathrm{Hg})\end{array}$ & Outcome \\
\hline $1 / 12$ & 57 & 13 & 16 & 130 & 70 & 130 & 60 & 120 & $\mathrm{CC}$ & 45 & 130 & $\mathrm{CC}$ \\
\hline $2 / 9$ & 21 & 12 & 14 & 120 & 70 & 120 & 65 & 120 & $\mathrm{CC}$ & 60 & 110 & $\mathrm{CC}$ \\
\hline $3 / 11$ & 39 & 10 & 12 & 100 & 55 & 110 & 60 & 120 & $\mathrm{CC}$ & 35 & 120 & CC \\
\hline $4 / 8$ & 23 & 10 & 12 & 95 & 60 & 105 & 55 & 100 & CC & 30 & 115 & CC \\
\hline $5 / 7$ & 26 & 11 & 14 & 100 & 60 & 110 & 55 & 105 & $\mathrm{CC}$ & 50 & 120 & $\mathrm{CC}$ \\
\hline $6 / 10$ & 37 & 10 & 12 & 100 & 55 & 100 & 55 & 100 & CC & 30 & 130 & CC \\
\hline $7 / 5$ & 19 & 7 & 9 & 100 & 60 & 115 & 50 & 110 & CC & 25 & 100 & CC \\
\hline \multicolumn{13}{|c|}{ Mean (SD) values } \\
\hline $8.9(2.4)$ & $31.7(13.6)$ & $9.8(1.7)$ & $11.4(1.8)$ & $106(13)$ & $\begin{array}{l}61(6) \\
p<0.05\end{array}$ & $112(10)$ & $\begin{array}{l}57(5) \\
p<0.05\end{array}$ & $110(9)$ & & $\begin{array}{l}37(9) \\
p<0.05\end{array}$ & $117(10)$ & \\
\hline
\end{tabular}

$\mathrm{p}$ Values relate to comparisons with SPAP 1.

AMVSDO, Amplatzer muscular ventricular septal defect occluder diameter; CC, complete closure; SAOP, systolic aortic pressure; SPAP 1, systolic pulmonary artery pressure before balloon occlusion; SPAP 2, systolic pulmonary artery pressure during balloon occlusion.

7-18 minutes). No patient experienced any complication as a result of the procedure.

\section{Follow up}

There was a further fall $(\mathrm{p}<0.05)$ in the mean systolic pulmonary artery pressure (to $37(9) \mathrm{mm} \mathrm{Hg}$ ) at the one year follow up cardiac catheterisation. No complications were observed in the early postprocedural period or during the one year follow up. All patients had complete closure with no evidence of device recanalisation, migration, wire fracture, thromboembolism, or endocarditis (fig 2). No obstruction of the left pulmonary artery or the aorta was noted. The patient with the PDA and the perimembranous ventricular septal defect had successful surgical closure of the latter one month after transcatheter closure of the duct.

\section{DISCUSSION}

Percutaneous closure using the new Amplatzer duct occluder has significantly improved the results of tanscatheter closure of moderate sized and large ducts. Its major advantages over previous devices are the smaller delivery sheaths (67 French), the ability to reposition the device before release, and a significantly lower rate of complications and residual shunts. ${ }^{8-10}$ However, the Amplatzer duct occluder-as well as the other currently available duct occluders such as the Gianturco coils, ${ }^{4-6}$ the buttoned device, ${ }^{7}$ and the Gianturco-Grifka vascular occluding device ${ }^{14}$ - are not designed to maintain a stable position under high pressure. Thus in the setting of high pulmonary artery pressure there is a real possibility of systemic embolisation. In fact, we know of two instances of embolisation of an Amplatzer duct occluder into the aorta (Amplatz K, personal communication). In both these cases there was a systemic, and apparently following occlusion a suprasystemic, pulmonary artery pressure (pulmonary hypertensive crisis).
The findings of our present study-which we believe describes the first clinical experience with the Amplatzer muscular ventricular septal occluder for closing persistent arterial ducts-indicate that transcatheter closure of large HPAP-PDAs is feasible, effective, and safe. Complete occlusion was obtained in all patients, with a significant fall in the pulmonary artery pressure and no complications during the procedure or at the one year follow up. The device used is a modified Amplatzer septal occluder which has all the previously described advantages of the Amplatzer duct occluder, while its retention disk system ensures secure positioning in the pulmonary orifice of the duct and prevents device embolisation into the systemic circulation in the presence of high pulmonary artery pressure. In addition, because of its construction from tightly woven Nitinol wire, the AMVSDO exerts an exaggerated stenting effect on the duct wall, giving it greater stability than the Amplatzer duct occluder. Finally, this occluding device is available in sizes up to $24 \mathrm{~mm}$, which makes it suitable for transcatheter closure of very large ducts.

Oversizing of the Amplatzer duct occluder has been reported to play an important role in achieving a virtually $100 \%$ occlusion rate. ${ }^{9}$ In our study the device was selected according to the balloon stretched diameter of the duct, which was $2-3 \mathrm{~mm}$ larger than the angiographically estimated diameter. This not only increases the efficacy of the procedure but also reduces the risk of embolisation by achieving better device fixation and stability across the duct channel.

In this series of patients pulmonary vascular resistance was not included among the criteria for proceeding to duct closure. We believe that balloon duct occlusion is a useful test to define the reversibility of pulmonary artery hypertension in HPAPPDA (systolic pulmonary artery pressure more than two thirds of the systemic pressure). A significant fall in pulmonary artery pressure during balloon duct occlusion indicates potentially reversible pulmonary artery hypertension. However,
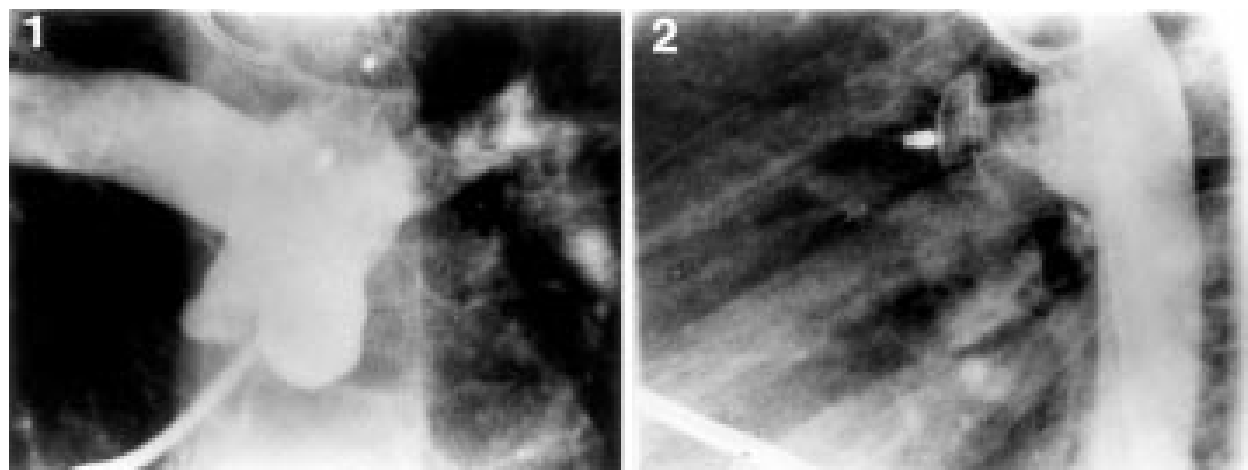

Figure 2 Pulmonary arteriogram (1) and descending aortogram (2) one year after implantation of the AMVSDO, showing complete closure and good position of the device with no evidence of aortic or pulmonary artery obstruction. 
evaluation of the reduction in the calculated pulmonary vascular resistance in response to oxygen or drug administration is recommended in cases of HPAP-PDA with fixed or nonsignificantly reduced pulmonary artery pressure during the balloon occlusion test.

\section{Study limitations}

While we successfully closed large ducts ranging from $7-13 \mathrm{~mm}$ in size and $6-8 \mathrm{~mm}$ in length with an Amplatzer muscular ventricular septal defect occluder, this device was not specifically designed for this purpose and has two limitations. Firstly, it is only available in one length, and ducts vary considerably in length. With very short ducts the device will extend too far into the pulmonary artery and may cause partial obstruction. Secondly, the retention disks are at a right angle but the insertion of the duct into the aorta and pulmonary artery is angled. ${ }^{9}$ In young children, therefore, the aortic disk may extend too far into the aorta and cause significant obstruction. A more ideal design would have been a prosthesis available in several lengths with two angled disks.

\section{Conclusions}

The AMVSDO is an important adjunct for closure of large ducts associated with high pulmonary artery pressure. Further studies are required to document its efficacy, safety, and long term results in a larger number of patients.

\section{Authors' affiliations}

B D Thanopoulos, G S Tsaousis, N G Eleftherakis, Department of Cardiology, Aghia Sophia Children's Hospital, Athens, Greece

M Diukic, S D Simeunovic, Department of Cardiology, University

Children's Hospital, Belgrade, New Yugoslavia

F Al Hakim, Department of Paediatric Cardiology, Queen Alia Heart Institute, Amman, Jordan

This study was presented at the World Congress of Pediatric Cardiology and Cardiac Surgery, Toronto, May 27-31, 2001.

\section{REFERENCES}

1 Rashkind WJ, Mullins CE, Helenbrand WE, et al. Non-surgical closure of patent ductus arteriosus: clinical application of the Rashkind PDA of patent ductus arteriosus: clinical application system. Circulation 1987;75:583-92.

2 Hosking MCK, Benson LN, Musewe N, et al. Transcatheter occlusion of the persistently patent ductus arteriosus: forty-month follow-up and prevalence of residual shunting. Circulation 1991;84:2313-17.

3 Verin VE, Saveliev VS, Kolody SM, et al. Results of transcatheter closure of the patent ductus arteriosus with the Botalloocluder. J Am Coll Cardiol 1993;22:1509-14

4 Hijazi ZM, Geggel RL. Results of anterograde transcatheter closure of patent ductus arteriosus using single or multiple Gianturco coils. J Am Coll Cardiol 1994;74:925-9.

5 Hijazi ZM, Geggel RL. Transcatheter closure of large patent ductus arteriosus ( $\geqslant 4 \mathrm{~mm}$ ) with multiple Gianturco coils: immediate and mid-term results. Heart 1996:76:536-40.

6 Uzun O, Hancock S, Parsons JM, et al. Transcatheter occlusion of the atrial duct with Cook detachable coil: early experience. Heart 1996;76:269-73.

7 Rao PS, Kim SH, Choi JY, et al. Follow-up of transvenous occlusion of patent ductus arteriosus with the buttoned device. J Am Coll Cardiol 1999;33:820-6.

8 Masura J, Kevin P, Thanopoulos B, et al. Catheter closure of moderateto large-sized patent ductus arteriosus using the new Amplatzer Duct Occluder: immediate and short-term results. J Am Coll Cardiol 1998;31:878-82.

9 Thanopoulos B(V)D, Hakim FA, Hiari A, et al. Further experience with transcatheter closure of the patent ductus arteriosus using the Amplatzer duct occluder. J Am Coll Cardiol 2000;35:1016-21.

10 Faella HJ, Hijazi ZM. Closure of the patent ductus arteriosus with the Amplatzer PDA device: immediate results of the international clinical trial. Cathet Cardiovasc Intervent 2000;51:50-4.

11 Thanopoulos B(V)D, Tsaousis GS, Konstadopoulou GN, et al. Transcatheter closure of muscular ventricular septal defects with the Amplatzer ventricular septal defect occluder: initial clinical application in children. J Am Coll Cardiol 1999;33:1395-9.

12 Hijazi ZM, Hakim F, Al-Fadley F, et al. Transcatheter closure of single muscular ventricular septal defects using the Amplatzer muscular VSD occluder: initial results and technical considerations. Cathet Cardiovasc Intervent 2000;49: 167-72.

13 Krichenko A, Benson LN, Burrows $P$, et al. Angiographic classification of the isolated, persistently patent ductus arteriosus and implications for percutaneous catheter occlusion. Am J Cardiol 1989:67:877-80.

14 Ebeid MR, Gaymes CH, Smith JC, et al. Gianturco-Grifka vascular occlusion device for closure of patent ductus arteriosus. Am J Cardiol $2001 ; 87: 657-60$.

\section{IMAGES IN CARDIOLOGY \\ Aortic abscess to right atrium fistula}

A previously fit and well 34 year old man was admitted with a six week history of increasing breathlessness and non-productive cough. He was febrile with splinter haemorrhages present and in cardiac failure. He had a wide pulse pressure and a long early diastolic murmur. The PR interval was prolonged at $300 \mathrm{~ms}$. A transthoracic echocardiogram confirmed severe aortic regurgitation secondary to an infected bicuspid aortic valve. Transoesophageal echocardiography (TOE) revealed a highly disorganised bicuspid aortic valve with mobile vegetations attached to both cusps and an aortic root abscess. Blood cultures subsequently grew Streptococcus viridans. The patient underwent an emergency aortic valve replacement with a Starr Edwards prosthesis since no homograft was available. The intraoperative findings were of an extensive pancarditis with a fibrinous pericardial effusion. The aortic valve was virtually destroyed by infection and the aortic abscess bulged into the right atrium, where the atrial wall was described as "paper thin". The patient started to make a good recovery early in the postoperative period but a week later he developed acute right heart failure. A repeat TOE (right) revealed a fistula from the original abscess cavity into the right atrium (AVR, aortic valve replacement). Despite aggressive diuretic treatment, he continued with symptomatic right ventricular failure secondary to overload. After completing a course of penicillin and gentamicin, the patient did not appear to be infected and an aortic to right atrium fistula repair was undertaken using a bovine

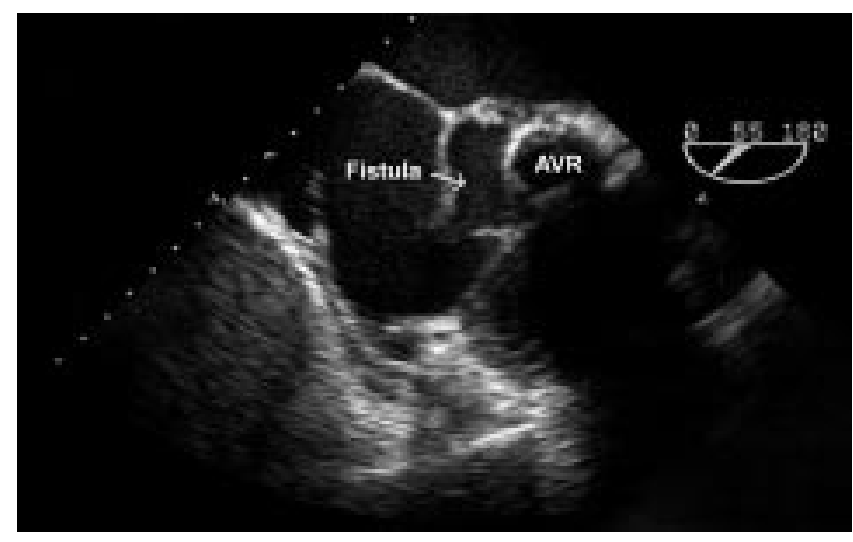

pericardial patch. The patient made a good recovery from his surgery.

P R James

L S Lee

S R M Holmberg rachael.james@virgin.net 